\title{
Xanthogranulomatous pyelonephritis in a horseshoe kidney
}

\section{Basson, J de Witt}

Division of Radiodiagnosis, Tygerberg Academic Hospital, Bellville, Western Cape

C Basson, MB ChB

J de Witt, MB ChB

Corresponding author: J de Witt (juruandewitt@gmail.com)

A rare case of xanthogranulomatous pyelonephritis in a horseshoe kidney is presented, with emphasis on the computed tomography findings. This very rare combination has only been reported twice in an adult, and never been published with images.

S Afr J Rad 2013;17(1):24-25. DOI:10.7196/SAJR.771

A rare case of xanthogranulomatous pyelonephritis (XGPN) in a horseshoe kidney is presented with emphasis on the computed tomography (CT) findings. This exceedingly rare combination has only been reported on two previous occasions in an adult ${ }^{[1]}$ and has never before been published with images. Radiographic findings are demonstrated in Fig. 1, while Figs 2 and 3 demonstrate some of the distinctive features seen on CT imaging.

Xanthogranulomatous pyelonephritis is an uncommon form of chronic pyelonephritis characterised by a destructive granulomatous

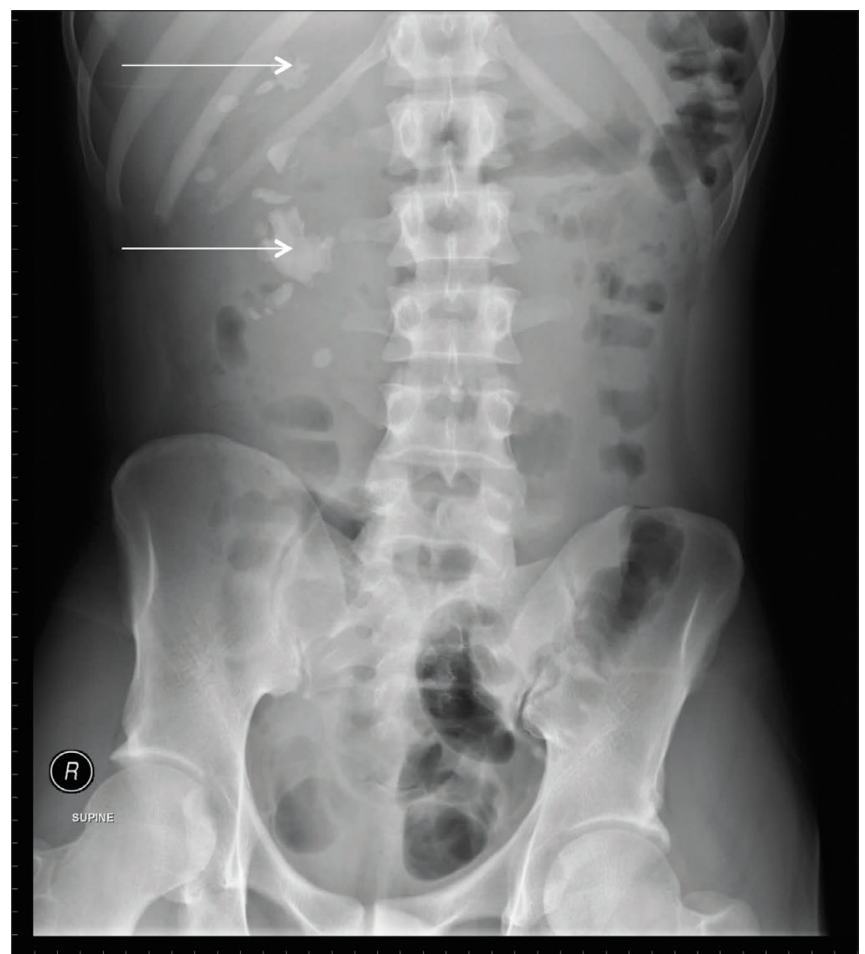

Fig. 1. Anterior X-ray demonstrating numerous well-defined densities (white arrows) projecting over the middle and upper aspects of the right abdomen. process of the renal parenchyma with abscess formation. XGPN occurs in approximately $1 \%$ of all renal infections. XGPN is four times more common in women than men, and is usually noted in the 5th and 6th decades of life. XGPN displays neoplasm-like properties capable of local tissue invasion and destruction, and is occasionally referred to as a pseudotumour ${ }^{[2]}$ Most cases occur in the setting of infected obstruction owing to renal calculi. The pathognomonic microscopic feature is that of lipid-laden, foamy macrophages; ${ }^{[3]}$ hence the prefix xantho-, from the Greek xanthos, meaning yellow.

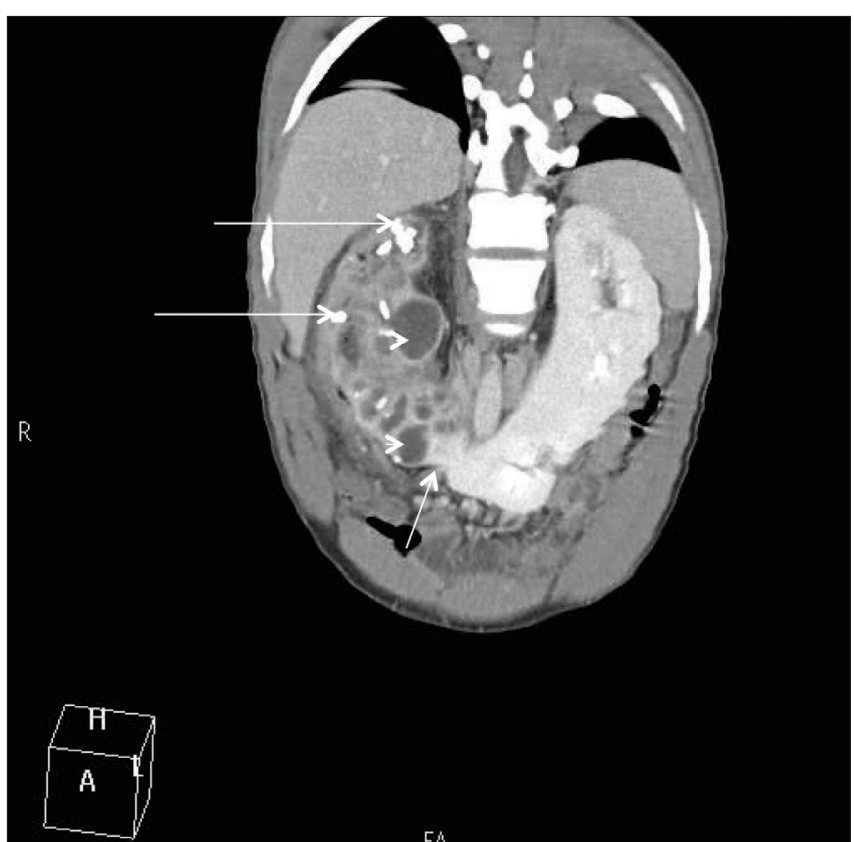

Fig. 2. Coronal reformatted CECT of the abdomen, confirming the densities to be right-sided renal calculi within a horseshoe kidney (long arrows). Note the isthmus, where the two kidneys are connected at their inferior poles (short arrow). Multiple focal hypo-attenuating lesions are scattered throughout the right aspect of the horseshoe kidney (arrowheads). 


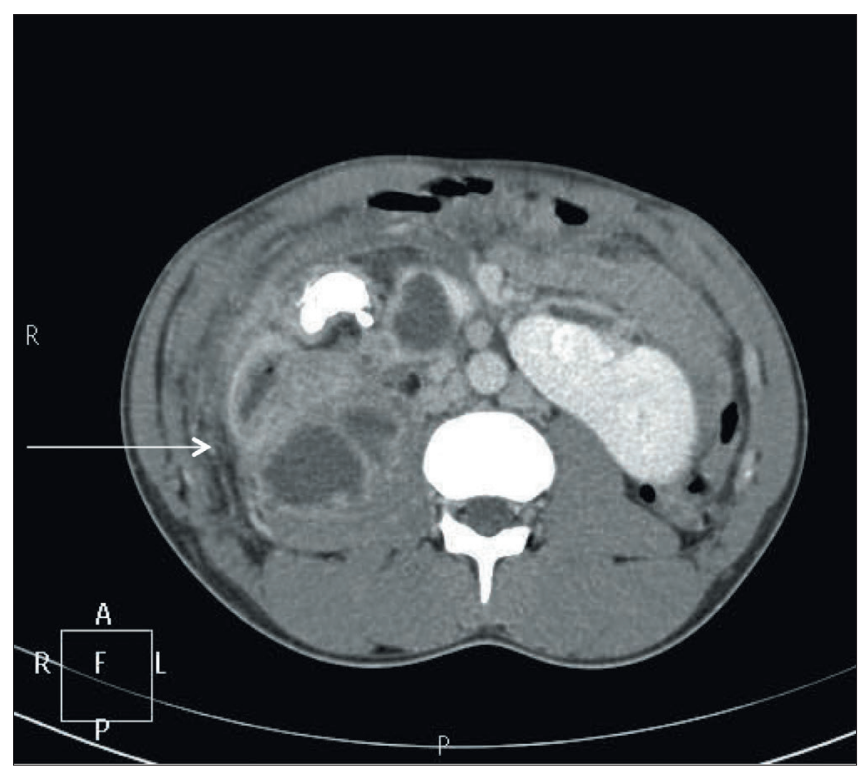

Fig. 3. Axial reformatted CECT of the abdomen revealing right-sided inflammatory perinephric fat stranding (arrow).

A horseshoe kidney represents the most common renal fusion anomaly, where the two kidneys are connected across the midline by an isthmus. Horseshoe kidneys are frequently complicated by obstruction, infection and calculus formation, and have an increased risk of renal malignancy development, especially Wilms' tumour.

\section{CT findings}

The combination of a nonfunctioning enlarged kidney, a central calculus within a contracted renal pelvis, expansion of the calyces, and inflammatory changes in the perinephric fat is strongly suggestive of XGPN. ${ }^{[4]}$ The contracted renal pelvis with dilated calyces has been likened to the pawprint of a bear; hence the diagnostic bear paw sign. ${ }^{[5]}$

Although the branching pattern of hypoattenuation that extends from the contracted renal pelvis may suggest hydronephrosis, it actually corresponds to an extensive inflammatory infiltrate rather than fluid in almost all cases. Renal function (excretion) is rarely seen at the time of diagnosis, although variable rim-enhancement surrounding the central branching inflammatory process is common.

The majority of cases demonstrate a highly specific set of findings that allows the radiologist to diagnose the condition confidently. Surgical planning depends on the accurate assessment of the extrarenal extent of disease, further supporting CT investigation as the imaging modality of choice.

1. Mongha R, Dutta A, Vijay M, Chatterjee U, Chakraborty SC. Xanthogranulomatous pyelonephritis in a horseshoe kidney. Saudi J Kidney Dis Transpl 2010;21:515-517.

2. Lorentzen M, Nielsen HO. Xanthogranulomatous pyelonephritis. Scand J Urol Nephrol 1980;14(2):193200 .

3. Learning Radiology.com. Xanthogranulomatous pyelonephritis. http://www.learningradiology.com/ archives2010/COW\%20393-XGPN/xgpncorrect.htm (accessed 18 July 2012).

4. Craig WD, Wagner BJ, Travis MD. Pyelonephritis: radiologic-pathologic review. Radiographics 2008;28(1):255-277.

5. Dyer RB, Chen MY, Zagoria RJ. Classic signs in uroradiology. Radiographics 2004;24(1):247-280. 